\title{
Research with Families Facing Cancer: The Challenges of Accrual and Retention*
}

\author{
Laurel L. Northouse, ${ }^{1 \dagger}$ Tansey Rosset,${ }^{1^{\ddagger}}$ Laurel Phillips, ${ }^{1 \dagger}$ \\ Darlene Mood, ${ }^{2 \uparrow}$ Ann Schafenacker, ${ }^{1 \#}$ Trace Kershaw $^{3 * *}$
}

\author{
${ }^{1}$ University of Michigan School of Nursing, Ann Arbor, Michigan \\ ${ }^{2}$ Wayne State University College of Nursing, Detroit, Michigan \\ ${ }^{3}$ Department of Epidemiology and Public Health, Yale University, New Haven, Connecticut \\ Accepted 3 February 2006
}

\begin{abstract}
The purposes of this article are: (a) to describe and analyze the accrual and retention patterns in a longitudinal randomized clinical trial with prostate cancer patients and their partners, and (b) to discuss strategies that were used to overcome challenges in conducting this family-based study. Initially, 429 dyads were referred to the study. Of these, 166 were not enrolled due to refusal $(n=120)$ or ineligibility $(n=46), 21$ of whom did not meet one or more of the inclusion criteria, and 25 of whom could not be reached within the 2-month window of eligibility. Of the 383 eligible dyads, 263 dyads were enrolled (enrollment rate of $68.7 \%$ ). Accrual and retention patterns differed by research site, referral procedures, and phase of prostate cancer. The retention rate was very good with the maiority of dyads $(n=218)$ completing all three follow-up assessments at 4, 8, and 12 months (82.9\%). (c) 2006 Wiley Periodicals, Inc. Res Nurs Health 29:199-211, 2006
\end{abstract}

Keywords: cancer; family; accrual; quality of life; retention, randomized clinical trial

There is growing recognition of the importance of family-based research. Families have been identified as the primary source of support to people with cancer, and they are the bedrock of chronic care providers in the United States (Arno, Levine, \& Memmott, 1999). Critical health decisions often take place within the family context and are seldom made by patients alone.

Contract grant sponsor: National Cancer Institute; Contract grant number: R01 CA 090739.

Correspondence to Laurel L. Northouse PhD, RN, FAAN, School of Nursing University of Michigan, 400 N. Ingalls, Ann Arbor, MI. 48109.

*We acknowledge the contributions of our outstanding data collectors: Charlotte Cowles, Andrea De Agostino, Marilyn Jeffs, Heather Kapp, Nancy Lunsford, Elizabeth Palen, Laurel Phillips, Tansey Rosset, Evelyn Sims, and Cordelia Tucker, as well as the excellent referral staff: Karen Baranowski, Monika Benedict, Judith Fardig, Kathleen Fasing, Elizabeth Galvin, Martha Heath, Patricia Kielczewski, Pamela Laszewski, Nancy Lefkowitz, Karin Olson, Donna Osborne, and Doris Sepe.

${ }^{\dagger}$ Mary Lou Willard French Professor of Nursing.

Proiect Research Nurse.

"Professor Emerita \& Senior Research Scientist.

"Project Manager FOCUS Program.

**Assistant Professor.

Published online in Wiley InterScience (www.interscience.wiley.com) DOI: 10.1002/nur.20128 
In spite of the family's integral role, little is known about the impact of cancer on the family or the experience of family caregivers. Family members seldom are included in research studies, and when they are, the study design and data collection procedures become more complex (McMillian \& Weitzner, 2003; Moriarty \& Cotroneo, 1993; Neumark, Stommel, Given, \& Given, 2001).

There are a number of difficulties that can hinder accrual and retention in family-based research, such as an extended recruitment phase with more people to contact and obtain consent, greater complexity in scheduling data collection sessions with multiple participants, possible inclusion of children, and at times, high refusal rates (Moriarty \& Cotroneo, 1993). However, in order to develop and test evidence-based interventions for patients and their families, investigators must overcome the challenges inherent in conducting family-based research.

The purposes of this article are: (a) to describe and analyze the accrual and retention patterns in a longitudinal randomized clinical trial for men with prostate cancer and their partners, and (b) to discuss strategies that were used to overcome challenges in conducting this family-based study.

Recruitment and retention of research subjects are generally considered to belong to the practical realm of scientific inquiry. However, the ability to successfully obtain and retain research subjects can have a profound effect on the study's validity and the ability to generalize study findings (Given, Keilman, Collins, \& Given, 1990). In addition, the use of power analyses to avoid Type II errors (i.e., falsely concluding that findings are not statistically significant) has placed further importance on accruing and retaining adequate numbers of subjects.

Gross, Mallory, Heiat, and Krumholz (2002) found that randomized clinical trial reports seldom include specific descriptions of the recruitment efforts for participants, making it difficult for readers to determine the generalizability of a study's findings. Recruitment and retention also represent a significant portion of the expense of conducting research (Motzer, Moseley, \& Lewis, 1997; Sears et al., 2003). It is common to underestimate the amount of time, money, planning, and organization necessary to recruit the desired number of subjects into a study (Swanson \& Ward, 1995). Furthermore, there may be a tendency for some investigators to trim their budgets unrealistically in areas related to accrual and retention as research funds are decreasing and competition for funding is increasing.
In trials of behavioral or psychosocial interventions, researchers have reported enrollment rates that range between 30 and $60 \%$ (Cooley et al., 2003). These percentages indicate that two or more potential participants must be referred for every participant who is subsequently enrolled in a study (Goodwin et al., 2000; Gross et al., 2002; Motzer et al., 1997; Sears et al., 2003). Many difficulties in enrollment have been reported in the literature including the following: the patient's degree of illness or fatigue; a lack of interest in the study purpose; lack of perceived benefit from the study; fear of harm from study participation; the complexity of the study; insufficient time; unwillingness to be randomly assigned into the experimental versus the control group or a strong preference for one of the two groups; demographic and socioeconomic factors; physician and nurse attitudes; and competing trials (Swanson \& Ward, 1995). In family-focused research, these problems multiply as enrollment becomes dependent on more than one person's willingness and ability to participate in the study (Moriarty \& Cotroneo, 1993).

Another problem that can hinder enrollment is the referral of persons who are thought to be eligible but who subsequently are determined to be ineligible when contacted by research personnel (McMillian \& Weitzner, 2003). This process uses up limited study resources because data collectors spend considerable time and effort trying to reach potential participants who, after the effort has been expended, do not meet preestablished study criteria.

Once an eligible participant has been successfully identified and enrolled, retention becomes the focus in longitudinal studies. While all longitudinal studies experience attrition, significant attrition over the course of the study may result in biased findings and can increase study costs (Given et al., 1990; Neumark et al., 2001). The attrition rates in longitudinal studies vary considerably, from 16 to $50 \%$, depending on the population studied (McMillian \& Weitzner, 2003; Moser, Dracup, \& Doering, 2000; Motzer et al., 1997). Reasons for attrition include the death of the subject, the demands of illness and treatments, the requirements or duration of the study, lack of time, loss of interest, subject frustration at not being randomized into the desired group, subject relocation, and loss of contact (Cooley et al., 2003; Pruitt \& Privette, 2001). In a study of a family intervention for women with breast cancer requiring the participation of the patient and her spouse, divorce or family dissolution also was reported as a cause for withdrawal (Motzer et al., 1997). 
While no single model or theory of recruitment and retention has been proposed, several researchers have identified key strategies that may benefit future researchers. Killien and Newton (1990) described several concepts that guided their recruitment and retention in a longitudinal study of coronary artery bypass patients and their spouses. These included establishing a philosophy of caring for participants, maintaining consistent contact with participants, and utilizing a plan for systematic follow-up between researchers and participants. McNees, Dow, and Loerzel (2005) reported a dramatic increase in enrollment when referring physicians were contacted to get a renewed commitment to the study, monthly enrollment reports were distributed to referring physicians, and research staff made weekly visits to the cancer center.

Given et al. (1990) conducted a longitudinal study of family caregivers for elderly and physically impaired patients and reported an attrition rate of less than $4.5 \%$, excluding death. The strategies employed included the following: in-depth preparation of data collectors; encouraging strong association with the study using project logos on letters and incentives; ongoing communication with the subjects through the use of letters, phone calls, and newsletters; maintaining continuity between the subject and the data collector; and expressing appreciation of subjects' participation with small gifts and/or monetary incentives.

This review of literature indicates that there are a number of factors that appear to affect accrual and retention in research studies and that certain strategies are associated with enhanced enrollment and retention. However, it is also evident from examination of the literature that many researchers do not discuss the strategies used to enhance accrual and retention in any substantive detail, and they seldom assess the utility of the few strategies they do report. This article examines accrual and retention patterns in a family-based randomized clinical trial, discusses the carefully planned strategies designed to facilitate those efforts, and reports on the results that support the utility of those strategies.

\section{METHODS}

\section{Study Design}

A longitudinal randomized clinical trial was designed to test the effects of a family intervention, called the FOCUS Program, on the quality of life and other psychosocial outcomes of men with prostate cancer and their spouses or partners. The intervention had been tested previously with dyads facing recurrent breast cancer (Northouse, Kershaw, Mood, \& Schafenacker, 2005). Men with prostate cancer and their spouses were assessed at baseline (T1), and then stratified by research site (three) and by phase of illness (three). Once stratified, dyads were randomized to the control condition (usual care) or experimental intervention (usual care plus the FOCUS Program). All dyads were reassessed at 4 months (T2), 8 months (T3), and 12 months (T4) from baseline.

\section{Sample}

Eligibility criteria. Men were eligible for the study if they were in one of three phases of prostate cancer: (a) newly diagnosed localized phase, (b) post-primary treatment phase with a rising prostate-specific antigen (PSA), indicating a biochemical recurrence, and (c) advanced/metastatic phase. Within each phase, patients may have had one or more treatments for their cancer. Patients in the localized phase received either a radical prostatectomy or external beam radiation. Patients with biochemical recurrence were under watchful waiting or some form of treatment (e.g., hormonal therapies). Patients with advanced cancer received a variety of types of chemotherapy or hormonal treatments. For each cancer phase, patients were eligible if they were within a 2-month window of eligibility: (a) following post-primary treatment (newly diagnosed phase), (b) following two successive rises in their PSA level (post-treatment rising PSA phase), or (c) following disease progression or change of treatment (advanced phase). The 2-month window of eligibility was used to obtain patients and family members who were dealing with a new illness, progression of their illness, or change of treatment and thus likely to derive more benefit from the planned intervention.

In addition, patients had to be 30 years of age or older, have a life expectancy of at least 12 months (assessed by physician), live within a 75 mile radius of one of the participating research sites, and have a spouse or live-in partner (male or female) who also was willing to be in the study. Spouses/partners were eligible if they were age 21 or older and identified by the patient as his spouse or partner. Patients were excluded if they had a second primary cancer, and spouses were excluded if they themselves had been diagnosed with cancer 
within the previous year or were receiving active treatment for cancer. The dyad was excluded if either member was ineligible.

Target sample. Based on power analysis (Cohen, 1988), the target sample for this study was 222 dyads (men with prostate cancer and their spouse/partner). From census data at our two initial research sites (both major comprehensive cancer centers), we anticipated that there would be a very large accessible pool of patients with prostate cancer from these two sites (see Table 1). Also based on available data, we projected that most of the accessible patients would be in the localized phase (54\%), with fewer patients in the post-treatment rising PSA phase $(15 \%)$ or the advanced/metastatic phase (31\%).

In the research planning stages, we carefully analyzed a number of factors that would be expected to lower the number of eligible men available from this accessible pool (see Table 1). We estimated that only $70 \%$ of the men would have a willing or able-to-participate spouse/ partner based on our previous family-focused studies (e.g., Northouse et al., 2005), and that only $75 \%$ of the men would live within a 75 -mile radius of the research sites. We also anticipated that while $95 \%$ of the men in the newly diagnosed localized group would have a life expectancy of at least 12 months (necessary to complete this longitudinal study), only $85 \%$ of the men in the posttreatment rising PSA group with biochemical recurrence, and $60 \%$ of the men in the advanced/ metastatic group would meet this criterion.

After taking these multiple factors into consideration, we estimated the number of men who met all of the eligibility criteria that would be available each month (see Table 1). Our anticipated enrollment rate was $50 \%$ of the eligible referrals. Our anticipated attrition rate (i.e., withdrawal or death) was $28.5 \%$ of the enrolled dyads overall $(8 \%$ at $\mathrm{T} 2,9.5 \%$ at $\mathrm{T} 3$, and $11 \%$ at T4). Our projection of attrition rates over time was based on prior attrition in our previous longitudinal studies (Northouse, Templin, Mood, \& Oberst, 1998; Northouse et al., 2005). Based on these estimates, target enrollment was set at 300 dyads in order to retain 222 dyads through all four-assessment times. Combining our anticipated number of monthly referrals (16-18 per month) and a $50 \%$ enrollment rate, we projected an average monthly accrual rate of 8-9 dyads for 36 months. Because we used conservative estimates based on this complex array of factors, we did not project further variation in accrual rates related to season, physician conference schedules, or other systemic variables.

\section{Procedures}

Referral process. Initially two large cancer centers were enlisted to obtain participants for the study. Research Site One was a large comprehensive cancer center located in a suburban setting, which serves as a major treatment center for cancer patients in the state. This site serves primarily Caucasian patients $(90 \%)$ and some minority patients (10\%). Research Site Two was a large comprehensive cancer center, located in an urban setting, with a large network of referring community physicians. This site has a smaller majority of Caucasian patients $(63 \%)$ and a larger number of minority patients (37\%), including about 30\% African-American patients. Research Site Three was added when Research Site Two experienced a series of organizational, financial, and personnel changes that resulted in a significant decrease in referrals. Research Site Three was a

\section{Table 1. Estimates of Factors That Affect Accrual}

\begin{tabular}{lccc}
\hline & \multicolumn{3}{c}{ Phase } \\
\cline { 2 - 4 } Estimates of Various Factors & $\begin{array}{c}\text { Newly Diagnosed } \\
\text { Localized }\end{array}$ & $\begin{array}{c}\text { Post-Treatment } \\
\text { Rising PSA }\end{array}$ & $\begin{array}{c}\text { Advanced/ } \\
\text { Metastatic }\end{array}$ \\
\hline Accessible pool of men with prostate cancer & 640 & 180 & 365 \\
Other eligibility criteria & 448 & 126 & 237 \\
Available spouse (70\%) & 336 & 94.5 & 178 \\
Live within 75 miles from site $(75 \%)$ & 319 & 80 & 107 \\
12-month life expectancy & 26.6 & 6.7 & 8.9 \\
Dyads available per month & 13.3 & 3.3 & 4.5 \\
Dyads if 50\% response rate & & & \\
\hline
\end{tabular}

a Estimates are $95 \%$ for Phase I, $85 \%$ for Phase II, 60\% for Phase III. PSA, prostate specific antigen. 
major hospital with a community-based cancer center, located in a suburban area. This site serves a majority of Caucasian cancer patients $(91 \%)$ and some minority patients $(9 \%)$.

At each research site, designated referral persons were identified who were responsible for screening clinic records, identifying eligible patients, and informing the patients about the research study. These referral persons were clinic staff nurses, nurse practitioners, or physician assistants in one of three types of clinics: radiation oncology, surgical oncology, or medical oncology. Each referral staff member was trained individually or in a group format about patient eligibility criteria and referral procedures. Training of referral staff took approximately 4 hours, using two group sessions. Follow-up phone and email contacts reinforced subject referral procedures. Referral staff received a study notebook (displaying project logo) that contained information on the study purpose, eligibility criteria, introductory scripts, introductory letters to participants, maps showing a radius of 75 miles from the cancer center, record-keeping sheets for logging their contact efforts and outcomes, and a laminated $3 \times 5$ card containing "Fast Facts"-essential study information that they could keep in their lab coat pockets.

Referral staff received reimbursement for their services in three different ways depending on the institution, referring physician, and staff member's preferences. Option one: we contracted to reimburse the employing institution for 5 or $10 \%$ of the referral staff member's full-time salary. Referral staff who screened for patients from multiple referring physicians received a $10 \%$ buyout, while staff who screened patients from one or two physicians received a 5\% buyout. This option was available at Research Sites One and Two. Option Two: some referral staff were paid directly for their referral services at an hourly rate, receiving compensation at the end of the month on a special payment form. This option was available only at Research Site One. Option Three: At Research Site Three, a subcontract with the institution's clinical trials office provided direct and indirect cost reimbursement for one of their employees to be assigned to a $50 \%$ position to screen for eligible patients from multiple physicians' practices at that site.

Referral staff gave eligible patients an introductory letter that briefly explained the study, asked if the patient would be willing to speak to a member of the research staff, and assured them that agreeing to be contacted implied no commitment to participate. The letter was signed by cancer center physicians who supported the study, including the patient's own physician. At the cancer center, if the patient agreed to learn more about the study, the clinic referral staff contacted the research project manager and gave her the patient's name, phone number, and best times to contact the dyad.

Each referral staff received a monthly update from the project manager indicating whether referred dyads agreed or refused to join the study. This feedback prevented referral staff from approaching dyads more than once. The principal investigator and project manager held periodic lunch meetings with referral staff at each site to keep them informed about study progress and to problem-solve any difficulties with the referral process.

Recruitment process. As referrals were received, the project manager assigned the dyad to one of the study's data collectors. The data collector called the dyad and explained the study in greater detail. In light of the increasing use of caller-ID monitoring, privacy managers, and answering machines, it was decided to list the data collector's name on the introductory letter to increase the likelihood that potential dyads would answer the phone when called by the person whose name they had been given.

The training of data collectors included how to use an introductory phone script, role-playing responses to commonly asked questions, and instruction in obtaining informed consent. New data collectors also accompanied at least two experienced data collectors on data collection visits to increase new data collectors' familiarity and accuracy in conducting the research interview prior to being assigned to their own caseload. Overall, data collectors received approximately 40 hours of training by the project manager and principal investigator.

After receiving the name of a potential participant from the project manager, data collectors were expected to contact the dyad as soon as possible, preferably within 1-2 days. Data collectors used a phone script that included a detailed description of the study, and they tried to call dyads at a time when both patient and spouse could be on the phone together. Data collectors recorded the number of attempts made to reach each dyad and the outcome of each contact with the patient and/or spouse (refused study, agreed to participate, delayed decision, or unable to reach). When a dyad declined participation in the study, data collectors recorded the date and any reason(s) they gave for their decisions; however, no additional data on the dyad were recorded per the Health 
Insurance Portability and Accountability Act (HIPAA) rules.

For difficult-to-reach dyads, a protocol was established that the data collector would make at least six calls within a 2 -week period. If the data collector still was unable to reach the dyad, a letter was sent explaining the data collector's efforts to reach them by phone and asking them to confirm if they were still interested in learning about the study. The dyad was asked to call the data collector or to return a prepaid postcard that indicated their preference to be contacted or not about the study.

Any dyads who refused participation in the study were entered into a database using a code number along with their reason for refusal. Refusal rates and reasons were discussed at monthly data collector staff meetings. Strategies to address reasons for refusal and ways to facilitate enrollment among future dyads also were discussed at monthly meetings.

Dyads who agreed to be in the study were scheduled for the first data collection visit in their home. Data collection protocols specified that interviews take place in the dyad's home or other convenient location to minimize the burden of participation in the study. The first data collection visit included the review and signing of consent forms, followed by administration of the survey instruments which were completed independently by the patient and spouse. All study procedures were approved by the university and agency institutional review boards.

Retention process. Because attrition (i.e., withdrawal from the study) is to be expected in any longitudinal trial, a number of strategies were implemented proactively to facilitate retention. After completing the baseline interview, dyads were mailed a letter informing them of their random assignment to treatment or control group. The mailing included a timeline indicating when follow-up interviews would occur and the name of the data collector and/or intervention nurse (treatment group only) who would be calling them. Data collectors were blinded to the group assignment of dyads. To maintain consistency and foster the relationship between the dyad and the research study, the same data collector conducted all four data collection interviews. After every visit the data collector sent the dyad a tailored form letter expressing appreciation for their continued participation and encouragement to complete the next set of surveys.

Intervention nurses were masters prepared nurses who received 40 hours of training to deliver the FOCUS intervention. They were notified by the project manager when a dyad was randomized to the experimental group after the initial data collection. The intervention nurse then contacted the dyad and scheduled the first FOCUS Program intervention session to be held in the dyad's home. Again, to maintain consistency and foster rapport, the same intervention nurse conducted the five FOCUS sessions, which included three face-toface meetings and two phone contacts over a 3 -month period of time. Upon completion of the FOCUS program, the intervention nurse reported to the project manager, who in turn notified the dyad's data collector that the dyad's 4-month data collection interview could be scheduled. The project manager also notified the data collector to arrange the 4-month interviews with dyads in the control group after the appropriate passage of time, thus keeping the data collectors blinded to the dyad's group assignment.

A series of incentives were used in this study. The incentives were given to the dyad during data collection visits so that the same protocol could be followed for both the treatment and control groups. After the first interview, the patient and family member each received a mug with the project logo as suggested by Given et al. (1990). A positive message "Helping others through research" was printed on the mug to reinforce participants' sense of altruism from participating in the study. Participants received a $3 \times 3$ clock with a small project logo printed on it following the second interview. It was expected that the clock would remind them of their ongoing participation in the study. A magnifying bookmark with project logo was given after the third interview, which was thought to be appropriate for these typically older dyads with decreasing vision. At the final interview, patients and partners received \$20 each for completing the final set of questionnaires.

Any dyad who withdrew from the study was entered into a $\log$ along with the reason for withdrawal. If withdrawal was due to death of the patient, the spouse/caregiver was offered the option of continuing in the study, but was not included in the major analyses of the data. Retention rates and reasons for withdrawal were discussed at monthly data collector meetings along with strategies to prevent attrition.

\section{Data Analysis}

Descriptive statistics were used to analyze data pertaining to accrual and retention. Content 
analysis was used to examine the reasons that dyads gave to data collectors for either refusing enrollment or for withdrawing from the study. Comparisons of anticipated participation and retention rates were made using one-sample Chisquare tests. In addition, differences in participation and retention between groups (e.g., phase of illness) were made using Chi-square tests. Baseline measures of selected demographic, health status, and psychosocial variables were correlated with retention/attrition status to identify predictors of attrition.

\section{RESULTS}

\section{Referral and Accrual Outcomes}

Over the 3 years of subject accrual, 429 dyads were referred to the study from the three research sites (see Fig. 1). Of the 429 dyads, 166 dyads were not enrolled in the study; 46 dyads were ineligible, and 120 dyads refused participation. Ineligibility reasons are listed in Table 2. The majority of ineligible dyads $(54.3 \%)$ could not be contacted by the data collector within the 2-month window of

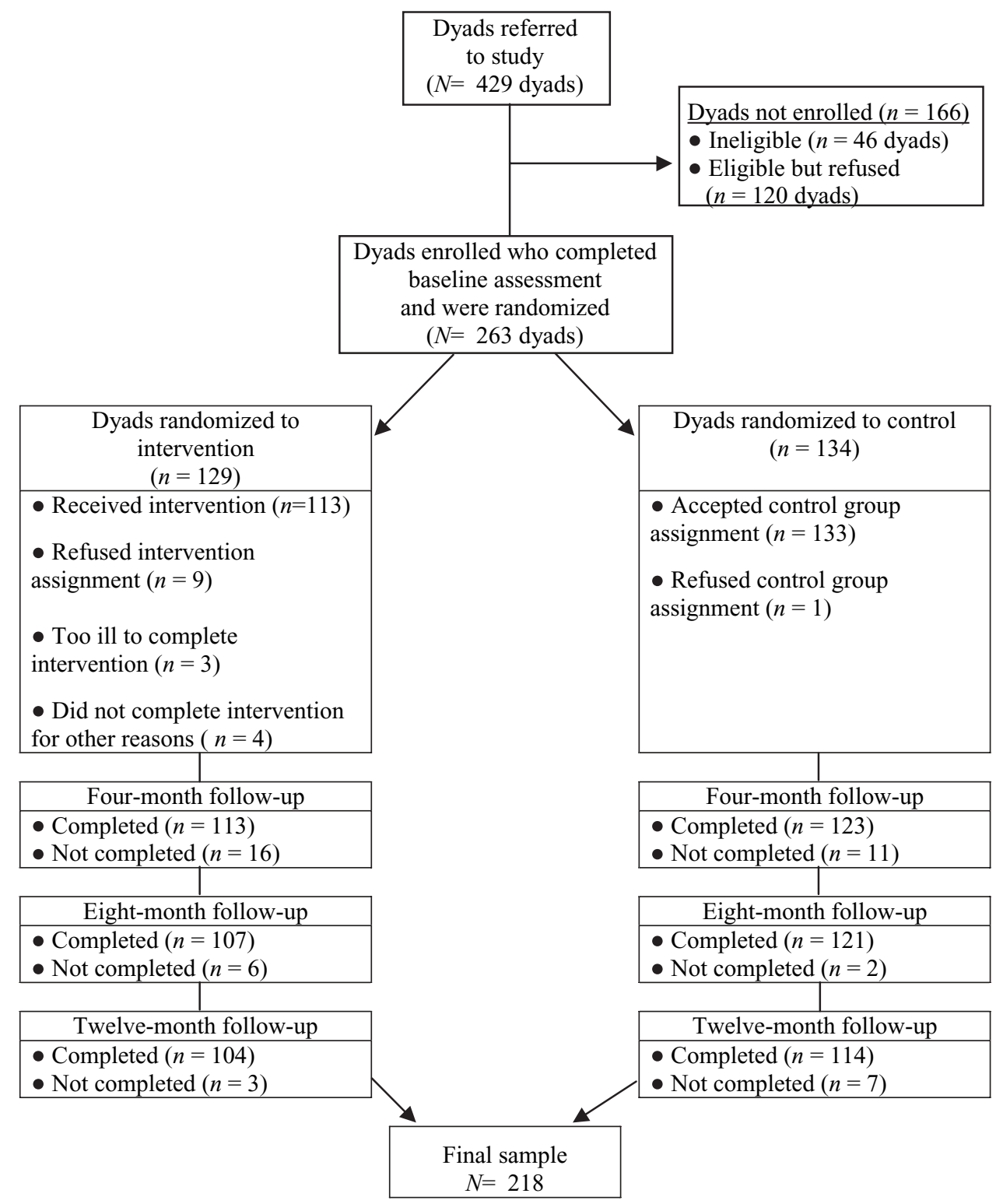

FIGURE 1. Flowchart Tracking Dyads Through Randomized Trial. 
Table 2. Data Pertaining to Dyads Not Enrolled in Study

\begin{tabular}{|c|c|c|c|}
\hline Referrals & $n$ & Reason $^{a, b}$ & $n$ by Subgroup \\
\hline \multirow[t]{4}{*}{ Ineligible } & 46 dyads $(27.7 \%)$ & $\begin{array}{l}\text { Could not contact within 2-month } \\
\text { window of eligibility }\end{array}$ & $25(54.3 \%)$ \\
\hline & & No spouse/partner & $9(19.6 \%)$ \\
\hline & & Has wrong type or more than one cancer & $3(6.5 \%)$ \\
\hline & & Other & $9(19.6 \%)$ \\
\hline \multirow[t]{8}{*}{ Eligible but refused } & 120 dyads ( $77.3 \%)$ & Patient not interested & $48(29.8 \%)$ \\
\hline & & Spouse not interested & $28(17.4 \%)$ \\
\hline & & Too busy & $37(23.0 \%)$ \\
\hline & & Study too personal & $11(6.8 \%)$ \\
\hline & & Too ill & $10(6.2 \%)$ \\
\hline & & Treatments too demanding & $5(3.1 \%)$ \\
\hline & & Plan frequent travel & $5(3.1 \%)$ \\
\hline & & Other & $17(10.6 \%)$ \\
\hline Total NOT Enrolled & 166 dyads $(100 \%)$ & & \\
\hline
\end{tabular}

${ }^{a}$ Could have more than one reason.

${ }^{\mathrm{b}}$ Reasons were previously established categories or new categories added after conversations with dyads.

eligibility even after multiple tries and by various methods (phone, mail). The remaining ineligible patients did not have a spouse/partner or did not meet one or more of the other inclusion criteria.

Content analysis of the reasons that dyads gave to data collectors when they refused participation is listed in Table 2. Interestingly, more patients than spouses reported they were not interested in being in the study, $\chi^{2}(1, n=120)=6.83$, $p<.001$. Other commonly given reasons were that the dyad was too busy, the study seemed too personal, or the patient was too ill. Taking into consideration the number of eligible referrals (383 dyads), and the number of refusals from the eligible dyads (120 dyads), the actual number of eligible dyads enrolled in the study was 263 , for an overall enrollment rate for this study of $68.7 \%$. This enrollment rate was significantly higher than the $50 \%$ that we projected in our study proposal, $\chi^{2}$ $(1, n=383)=14.40, p<.001$.

Data collectors kept a log of the number and outcome of every phone contact they made with each dyad until the dyad agreed to participate, refused to participate, or was found ineligible. We did a sub-sample analysis of the first 48 dyads referred to the study and found that it took 227 calls to these 48 dyads $(m=4.7$ calls per dyad) to enroll 38 of them in the study. The remaining 10 dyads were not enrolled in the study because they were ineligible or refused participation. On the average, it took 4.4 calls to reach a dyad who agreed to be in the study and 6 calls to reach a dyad who declined to be in the study. Of the 227 calls, $106(46.7 \%)$ resulted in an answering machine, privacy manager, busy signal, or no answer. Most of the calls that successfully reached a member of the dyad occurred after 5 PM in the evening $(42.9 \%)$ or in the early afternoon between $1 \mathrm{PM}$ and 5 PM (32.1\%). Fewer dyads were reached during morning hours between $9 \mathrm{AM}$ and 1 PM $(25 \%)$.

Table 3 lists the accrual of dyads by research site. Although we anticipated that Research Site One and Two would have a similar number of referrals, this was not the case. Research Site Two provided less than half of the eligible referrals

Table 3. Accrual by Research Site and Phase of IIIness

Dyads Enrolled by Phase of IIIness

\begin{tabular}{lcccccc}
\cline { 4 - 6 } Research Site & $\begin{array}{c}\text { Eligible } \\
\text { Referrals }\end{array}$ & $\begin{array}{c}\text { Enrolled } \\
\text { Dyads }\end{array}$ & $\begin{array}{c}\text { Enrollment } \\
\text { Rate }\end{array}$ & $\begin{array}{c}\text { Newly } \\
\text { Diagnosed } \\
\text { Localized }\end{array}$ & $\begin{array}{c}\text { Post-Treatment } \\
\text { Rising PSA }\end{array}$ & Advanced \\
\hline Site 1 & 257 dyads & 186 & $72.3 \%$ & $114(61.3 \%)$ & $29(15.6 \%)$ & $43(23.1 \%)$ \\
Site 2 & 104 dyads & 67 & $64.4 \%$ & $49(73.1 \%)$ & $4(6.0 \%)$ & $14(20.9 \%)$ \\
Site 3 & 22 dyads & 10 & $45.5 \%$ & $8(80.0 \%)$ & $0(0.0 \%)$ & $2(20 \%)$ \\
Total & 383 dyads & 263 & $68.7 \%$ & $171(65.0 \%)$ & $33(12.6 \%)$ & $59(22.4 \%)$ \\
\hline
\end{tabular}


expected from that site. Midway into the study the number of referrals from Research Site Two decreased because of organizational changes and fiscal problems, resulting in the departure of key referral physicians from that institution, and subsequently fewer referrals from that site. However, even when eligible dyads were referred to the study from this site, the enrollment rate was lower than at Research Site One, $\chi^{2}(2$, $n=383)=8.02, p<.05($ see Table 3$)$.

Research Site Three was opened during the final year of accrual to supplement low accrual at Research Site Two. As indicated on Table 3, the number of dyads enrolled from Research Site Three was low and the enrollment rate at this site was lowest of all of the sites. This may have been due in part to the short time that this site was open (i.e., 8 months) and to the different referral process used at this site. At this site, an employee of the Clinical Trials Office was hired to identify and inform dyads about the study. This employee was less likely to known by the patients. In contrast, at the other two sites, dyads were approached by direct care providers (nurse practitioners and physician assistants) who worked in referring clinics with the patients.

Table 3 also lists the enrollment of dyads by the patient's phase of prostate cancer. As anticipated, the majority of patients enrolled in the study were in the newly diagnosed, localized phase of illness (65\%), followed by the advanced phase $(22.4 \%)$ and the post-treatment rising PSA phase (12.6\%). We enrolled $11 \%$ more men in the newly diagnosed, localized phase than anticipated, $8.6 \%$ fewer men in the advanced phase, and $2.4 \%$ fewer men in the post-treatment rising PSA phase than projected. However, the rank order of enrollment by phase was as expected. Although we had clearly designated referral sources for the newly diagnosed (urological surgery) and advanced phases (urologic oncology), it was difficult to identify patients in the post-treatment risingPSA phase because they were often being followed by a variety of physicians in different settings.

Of the 263 dyads enrolled in the study, the average age for patients was 63.1 years $(S D$ 9.1) and for spouses was 59 years ( $S D$ 9.6). Both patients and spouses were well-educated averaging 15.6 years $(S D$ 3.4) and 14.8 years $(S D 2.7)$ of formal education, respectively. The racial breakdown was the same for patients and spouses: $84 \%$ were Caucasian, $15 \%$ African-American, and $1 \%$ other minority or multiracial. Approximately half of the dyads (53\%) reported family incomes above $\$ 75,000$, a little more than a third
$(37 \%)$ were in the $\$ 30-74,999$ range, and the remaining (10\%) had incomes less than $\$ 30,000$ a year.

These factors also were examined, comparing the three research sites. Significant differences were found between the three research sites in regard to patients' education, household income, and race. The differences were primarily between Research Site Two located in the urban setting and the other two sites. At Research Site Two, 22\% of the patients had incomes less than $\$ 30,000$ a year, whereas at Research Site One only $6 \%$ and at Site Three no patients reported an income at this lower level, $\chi^{2}(4, n=263)=14.80, p<.03$. Patients at Research Site Two had a mean of 14.4 (SD 3.3) years of formal education compared to 16.1 ( $S D$ $3.3)$ years at Site One and $15.9(S D 2.5)$ years at Site Three, $F(2,260)=6.13, p=.002$. In regard to race, $43.3 \%$ of the patients were minority at Research Site Two compared to $6.5 \%$ at Site One and $11 \%$ at Site Three, $\chi^{2}(4, n=263)=50.8$, $p<.001$. The racial distribution of our sample was similar to that anticipated for each of the three research sites, suggesting that accrual procedures produced a sample consistent with the populations from which they were drawn.

\section{Retention Outcomes}

Table 4 lists the overall retention rate and the retention rates by research site. Across all sites the overall retention rate was $82.9 \%$ for dyads who completed all four assessments over the 12 months. This retention rate was higher than the $71.5 \%$ anticipated for this study, $\chi^{2}$ (1, $n=263)=16.74, p<.001$. In regards to retention rates by research site, Research Site One had the highest retention rate $(86 \%)$, followed by Site Three and Site Two (see Table 4).

Not only was the retention rate higher than expected, but also there was an interesting, but unexpected, pattern of attrition by data collection

Table 4. Retention Rates by Research Site

\begin{tabular}{|c|c|c|c|}
\hline & $\begin{array}{c}\text { Enrolled } \\
\text { Dyads }\end{array}$ & $\begin{array}{l}\text { Retained } \\
\text { Dyads }\end{array}$ & $\begin{array}{l}\text { Percent Dyads } \\
\text { Retained }\end{array}$ \\
\hline Research Site & $n$ & $n$ & $\%$ \\
\hline Site 1 & 186 & 160 & $86.0 \%$ \\
\hline Site 2 & 67 & 50 & $74.6 \%$ \\
\hline Site 3 & 10 & 8 & $80.0 \%$ \\
\hline $\begin{array}{l}\text { Total } \\
\text { across sites }\end{array}$ & 263 & 218 & $82.9 \%$ \\
\hline
\end{tabular}


Table 5. Attrition Rates by Group Assignment and Data Collection Session

\begin{tabular}{|c|c|c|c|c|c|}
\hline \multirow[b]{2}{*}{ Group } & \multirow{2}{*}{$\begin{array}{c}\text { Dyads enrolled } \\
\text { and randomized } \\
\text { Time 1, } \\
\text { Baseline }\end{array}$} & \multicolumn{3}{|c|}{ Attrition by Session } & \multirow[b]{2}{*}{$\begin{array}{c}\text { Total } \\
\text { Attrition }\end{array}$} \\
\hline & & $\begin{array}{l}\text { Time 2, } \\
4 \text { Months }\end{array}$ & $\begin{array}{l}\text { Time } 3, \\
8 \text { Months }\end{array}$ & $\begin{array}{c}\text { Time 4, } \\
12 \text { Months }\end{array}$ & \\
\hline & $n$ & $n(\%)$ & $n(\%)$ & $n(\%)$ & $n(\%)$ \\
\hline Experimental ${ }^{a}$ & 129 & $16(12.4 \%)$ & $6(4.6 \%)$ & $3(2.3 \%)$ & $25(19.4 \%)$ \\
\hline Control & 134 & $11(8.2 \%)$ & $2(1.5 \%)$ & $7(5.2 \%)$ & $20(14.9 \%)$ \\
\hline Total & 263 & $27(10.3 \%)$ & $8(3.0 \%)$ & $10(3.8 \%)$ & $45(17.1 \%)$ \\
\hline
\end{tabular}

${ }^{a}$ Intervention occurred between baseline (Time 1) and 4 months (Time 2).

session (see Table 5). The attrition was highest $(10.3 \%)$ between baseline (Time 1) and 4 months (Time 2) after dyads were randomized to intervention or control group. The attrition during this period of time was $12.4 \%$ for dyads in the intervention group versus $8.2 \%$ for dyads in the control group. Although higher, this was not significantly different, $\chi^{2}(1, n=263)=2.33$, $p=.13$. Attrition at the remaining follow-up data collection sessions was fairly low for dyads in the intervention and control group, averaging around $3 \%$ for both groups combined. By the final followup data collection session at 12 months, attrition rate was $19.4 \%$ for dyads in the intervention group and $14.9 \%$ for dyads in the control group, which was not significantly different, $\chi^{2}(1$, $n=263)=0.92, p=.34$.

Table 6 provides the attrition rates for dyads according to patients' phase of illness and the content analysis of the reasons dyads gave for withdrawing from the study. Of the 45 dyads who withdrew, close to half were from the newly diagnosed localized phase $(46.7 \%)$, and the other half were from the advanced phase $(48.9 \%)$. However, the percent of dyads who withdrew from these two phases of illness, compared to the total number of dyads in each phase, was significantly different, $\chi^{2}(2, n=263)=22.5$, $p<.001$. More specifically, in the localized phase, which had 171 enrolled dyads, the attrition was $12.3 \%$. In the advanced phase, which had only 59 enrolled dyads, the attrition rate was $37.3 \%$. The reasons for attrition also differed between dyads in these two phases of illness. In the localized phase, dyads more commonly declined their random assignment to the intervention, said they did not need the study, or said they were too busy (see Table 6). In the advanced phase, the death of the patient and progression of the patient's illness were the most common reasons for attrition. We had planned for a lower retention rate among patients in the advanced phase and the actual retention rate $(62.7 \%)$ was close to our expectation $(60 \%)$. There were only two withdrawals

Table 6. Withdrawal by Phase of IIIness and Reason ${ }^{a}$

\begin{tabular}{|c|c|c|c|c|}
\hline \multirow[b]{2}{*}{ Reason } & \multicolumn{3}{|c|}{ Phase of illness } & \multirow[b]{2}{*}{ Total $n \%$} \\
\hline & $\begin{array}{l}\text { Newly Diagnosed } \\
\text { Localized }\end{array}$ & $\begin{array}{l}\text { Post-Treatment } \\
\text { Rising PSA }\end{array}$ & Advanced & \\
\hline 1. Declined intervention group & 8 & 0 & 1 & $9(20.0 \%)$ \\
\hline 2. Disliked completing questionnaires & 1 & 0 & 0 & $1(2.2 \%)$ \\
\hline 3. Too busy & 4 & 0 & 2 & $6(13.3 \%)$ \\
\hline 4. Illness progression & 1 & 0 & 2 & $3(6.7 \%)$ \\
\hline 5. Marital problems & 1 & 0 & 1 & $2(4.5 \%)$ \\
\hline 6. Lost to follow-up & 4 & 0 & 0 & $4(8.9 \%)$ \\
\hline 7. Patient died & 0 & 1 & 14 & $15(33.3 \%)$ \\
\hline 8. Study not relevant & 2 & 0 & 1 & $3(6.7 \%)$ \\
\hline 9. Declined control group & 0 & 0 & 1 & $1(2.2 \%)$ \\
\hline 10. Developed new health problem (stroke) & 0 & 1 & 0 & $1(2.2 \%)$ \\
\hline Total & 21 & 2 & 22 & $45(100 \%)$ \\
\hline
\end{tabular}

${ }^{a}$ Reasons were previously established categories or new categories added after conversations with dyads. 
in the post-treatment rising PSA group (see Table 6).

To assess factors related to attrition, we examined the relationships between baseline variables and subsequent attrition. Dyads who entered the study with more health problems (i.e., higher symptom distress, more disease uncertainty, and lower physical quality of life) were more likely not to complete the study.

\section{DISCUSSION}

Although there is considerable interest in randomized trials to further evidenced-based practice, the resources required to obtain and retain participants in these studies is often underestimated. In this study we proactively considered a complex array of factors, estimated accrual rates conservatively, and used a number of strategies that enabled us to accrue and retain a large number of dyads into a longitudinal randomized clinical trial.

\section{Accrual Issues}

Even though we were able to recruit a large number of dyads, with enrollment rates similar to or higher than other longitudinal studies involving cancer patients and families (Cooley et al., 2003; Giesler et al., 2005; McMillian \& Weitzner, 2003; Motzer et al., 1997), the process was not easy. We used two large research sites and added a third site when referrals from Research Site Two decreased considerably. It is possible that referrals were higher from Research Site One because of a large eligible pool of participants with more resources (i.e., higher education and income), stability within the organization, a greater number of key referral physicians, and flexibility in payment options for referral staff. Although we used three reimbursement options for referral staff, we cannot state with certainty that one option was superior to another. Regardless of the reimbursement option used, referral staff reported at times that they were overwhelmed with multiple clinic demands, had an influx of very ill patients, and were unable to screen consistently for eligible dyads.

It took data collectors longer than anticipated to contact potential dyads who were referred to the study. Nearly half of the calls made to potential participants resulted in an answering machine, privacy manager, or no answer. It was necessary for data collectors to make multiple calls to enroll a single dyad and even more calls to reach dyads who subsequently refused participation, despite the fact that referral staff obtained the "best time to call" information from each dyad, and the data collector's name was made known to the dyad in advance. These calls, along with the mail contacts, added to the time needed for accrual and used additional grant resources.

There were a number of dyads who became ineligible because they could not be contacted by data collectors within the 2-month window of eligibility. The 2-month window was initially established to enroll prostate cancer patients and spouses at times when patients were experiencing a change in their health status such as a new diagnosis, post-treatment rising PSA, or progression of the prostate cancer. In retrospect, however, this short 2-month window of eligibility, in conjunction with the greater time needed to reach dyads, may have been too restrictive. Among the dyads considered ineligible, there were few who did not meet other eligibility criteria. This suggests that referral staff were well informed about study criteria and for the most part accurately identified eligible dyads.

Among the eligible dyads that refused the study, refusals were somewhat greater among the patients than their spouses. Because all of the patients were male and nearly all the spouses were female, it is difficult to determine if the number of refusals was more a function of role (i.e., patient vs. spouse) or gender (male vs. female). However, anecdotal reports from data collectors suggest that the male patients often said they "did not need the study," "were not interested in talking about the cancer," or that their "cancer was gone." Even though we were able to enroll a large number of male patients and their spouses in the study, there may be a subgroup of male patients who prefer not to participate in studies of this type.

\section{Retention Issues}

The overall retention of dyads in this study was very good and significantly better than anticipated due in part to careful tracking of dyads during all phases of the study, clear protocols on how and when to follow-up with dyads, and well-trained research staff. A few factors affected retention in this study. The most obvious factor was phase of illness. More patients with advanced prostate cancer died during the course of illness than did patients in either of the other two phases, contributing to their higher attrition rate. The reasons for withdrawal also differed by phase of 
illness. Dyads in the localized phase withdrew because they did not want to be in the intervention, disliked the questionnaires, or were too busy. Dyads in the other two phases were more likely to withdraw due to other health problems, disease progression, or death.

Group assignment to experimental or control group also affected retention. There was slightly more attrition overall among dyads in experimental versus the control group, but the difference was not significant. This difference was more pronounced during the interval just following randomization (experimental group $=12.4 \%$, control group $=8.2 \%$ ) with minimal attrition from either group at 8 and 12 months follow-ups. Although we had anticipated that attrition would increase over the course of this longitudinal study (i.e., $8 \%, 9.5 \%$, and $11 \%$, respectively), due to the demands of a longitudinal study or to an increase in patient death over time, this was not the case. Some researchers have reported more attrition from the control group versus experimental group due to random assignment (Given et al., 1990; Pruitt \& Privette, 2001); however, only one dyad from the control group withdrew from this study for this reason. It is possible that the demands of a five-session intervention for the experimental group, in combination with four data collection times required of all participants, may have been too time consuming, especially for men in the localized phase who were often busy maintaining work and family schedules.

By examining baseline factors, we found that certain dyads were less likely to complete the study. Those were dyads in which the patient was sicker at the start of the study (more symptom distress, lower quality of life, and more advanced disease), and in which there was more uncertainty about the illness.

\section{Lessons Learned}

We learned a great deal about the practical aspects of scientific inquiry as we conducted this randomized clinical trial. First, we learned how important it is to consider the multiple factors that can affect study accrual and retention $a$ priori when planning a study. Too often researchers focus only on the accessible pool and do not make allowances for other eligibility criteria. One of the unique aspects of this study was that we carefully estimated how factors such as patients' phase of illness, potential life expectancy, availability of a spouse caregiver, and dyads' distance from research site would affect our accessible pool of participants. We also took into consideration realistic enrollment and retention rates, used various payment options for referral staff, and provided incentives relevant to the typically older dyads in the study. We learned, however, that even with this careful planning, the organizational environment at the institution, time demands on referral staff, as well as some socio-demographic factors, can affect the number of participants referred to the study.

Second, this study underscored the need for meticulous organization within a study, the value of careful monitoring of recruitment and retention, and the importance of training all research staff (referral, data collection, and intervention). These quality control measures were essential to our ability to successfully accrue and retain dyads in this longitudinal study. Future investigators need to be sure to plan adequate budgetary resources for monitoring recruitment and retention of subjects, and for training all research staff.

Third, we learned the value of fostering support among the various teams (referral staff, data collectors, intervention nurses) associated with the study. Monthly team meetings provided an opportunity for staff to learn new strategies from one another and to receive reassurance that many of the problems they encountered in their research role (e.g., reaching dyads with privacy managers, etc.) were shared by other staff and not a result solely of their own inexperience.

Fourth, we learned the value of using procedures that decreased participant burden and increased their attachment to the study. All data collection and intervention sessions (with the exception of phone follow-up calls) took place in the home at a time that was most convenient for patients and spouses (i.e., evening, weekend). Although home visits are more expensive, they seemed worth the cost as we had very little missing data, were able to observe participant fatigue, clarify confusing questions (based on verbal and nonverbal observations), assist participants with reading difficulties, and insure that patients and spouses completed questionnaires independently.

In summary, this study describes the successful accrual and retention among dyads in a familybased randomized clinical trial. A number of strategies were used to accurately assess the available pool of participants, facilitate enrollment, and retain dyads in this longitudinal study. The more investigators are able to anticipate the demands and utilize active strategies to facilitate accrual and retention, the more likely they will be to complete family-based studies successfully. 


\section{REFERENCES}

Arno, P.S., Levine, C., \& Memmott, M.M. (1999). The economic value of informal caregiving. Health Affairs, 18, 182-188.

Cohen, J. (1988). Statistical power analysis for the behavioral sciences, (2nd ed.) Hillsdale, NJ: Lawrence Erlbaum.

Cooley, M.E., Sarna, L., Brown, J.K., Williams, R.D., Chernecky, C., Padilla, G., et al. (2003). Challenges of recruitment and retention in mulitsite clinical research. Cancer Nursing, 26, 376-384.

Giesler, R.B., Given, B.A., Given, C.W., Rawl, S., Monahan, P., Burns, D., et al. (2005). Improving the quality of life of patients with prostate carcinoma. Cancer 104, 752-762.

Given, B.A., Keilman, L.J., Collins, C., \& Given, C.W. (1990). Strategies to minimize attrition in longitudinal studies. Nursing Research, 39, 184-186.

Goodwin, P.J., Leszcz, M., Quirt, G., Koopmans, J., Arnold, A., Dohan, E., et al. (2000). Lessons learned from enrollment in the BEST study-A multicenter, randomized trial of group psychosocial support in metastatic breast cancer. Journal of Clinical Epidemiology, 53, 47-55.

Gross, C.P., Mallory, R., Heiat, A., \& Krumholz, H.M. (2002). Reporting the recruitment process in clinical trials: Who are these patients and how did they get here? Annals of Internal Medicine, 137, 10-16.

Killien, M., \& Newton, K. (1990). Longitudinal research-The challenge of maintaining continued involvement of participants. Western Journal of Nursing Research, 12, 689-692.

McMillian, S.C., \& Weitzner, M.A. (2003). Methodologic issues in collecting data from debilitated patients with cancer near end of life. Oncology Nursing Forum, 30, 123-129.

McNees, P., Dow, K., \& Loerzel, V. (2005). Application of the CuSum Technique to evaluate changes in recruitment strategies. Nursing Research, 54, 399405.
Moriarty, H.J., \& Cotroneo, M. (1993). Sampling issues and family research: Recruitment and sampling strategies. In S. Feetham, S.B. Meister, J.M. Bell \& C.L. Gillis (Eds.), The Nursing of Families (pp. 7989). Newbury Park, NJ: Sage.

Moser, D.K., Dracup, K., \& Doering, L.V. (2000). Factors differentiating dropouts from completers in a longitudinal, multicenter clinical trial. Nursing Research, 49, 109-116.

Motzer, S., Moseley, J.R., \& Lewis, F.M. (1997). Recruitment and retention of families in clinical trials with longitudinal designs. Western Journal of Nursing Research, 19, 314-333.

Neumark, D.E., Stommel, M., Given, C.W., \& Given, B.A. (2001). Research design and subject characteristics predicting nonparticipation in a panel survey of older families with cancer. Nursing Research, 50, 363-368.

Northouse, L., Kershaw, T., Mood, D., \& Schafenacker, A. (2005). Effects of a family intervention on the quality of life of women with recurrent breast cancer and their family caregivers. Psycho-Oncology, 14, 478-491.

Northouse, L.L., Templin, T., Mood, D., \& Oberst, M. (1998). Couples' adjustment to breast cancer and benign breast disease: A longitudinal analysis. Psycho-Oncology, 7, 37-48.

Pruitt, R., \& Privette, A.B. (2001). Planning strategies for the avoidance of pitfalls in intervention research. Journal of Advanced Nursing, 35, 514-520.

Sears, S., Stanton, A.L., Kwan, L., Krupnick, J.L., Rowland, J., Meyerowitz, B.E., et al. ( 2003). Recruitment and retention challenges in breast cancer survivorship research: Results from a multisite, randomized intervention trial in women with early stage breast cancer. Cancer Epidemiology, Biomarkers \& Prevention, 12, 1087-1090.

Swanson, G.M., \& Ward, A.J. (1995). Recruiting minorities into clinical trials: Toward a participant friendly system. Journal of the National Cancer Institute, 87, 1747-1759. 